\title{
Adaptation and validation of the Gambling Motives Questionnaire-Financial (GMQ-F) in a sample of French-speaking gamblers
}

\section{Gaëtan Devos, Gaëlle Challet-Bouju, Jonathan Burnay, Pierre Maurage, Marie Grall-Bronnec \& Joël Billieux}

To cite this article: Gaëtan Devos, Gaëlle Challet-Bouju, Jonathan Burnay, Pierre Maurage, Marie Grall-Bronnec \& Joël Billieux (2016): Adaptation and validation of the Gambling Motives Questionnaire-Financial (GMQ-F) in a sample of French-speaking gamblers, International Gambling Studies

To link to this article: http://dx.doi.org/10.1080/14459795.2016.1264080

\section{Published online: 21 Dec 2016.}

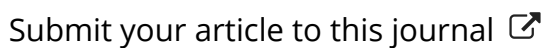

\section{Q View related articles $\sqsubset$}

View Crossmark data $₫$ 


\title{
Adaptation and validation of the Gambling Motives Questionnaire-Financial (GMQ-F) in a sample of French- speaking gamblers
}

\author{
Gaëtan Devos ${ }^{a}$, Gaëlle Challet-Boujub,c (D), Jonathan Burnay ${ }^{d}$, Pierre Maurage ${ }^{a}$, \\ Marie Grall-Bronnec ${ }^{b, c}$ and Joël Billieux ${ }^{a, e}$ \\ aLaboratory for Experimental Psychopathology (LEP), Psychological Sciences Research Institute, Université \\ catholique de Louvain, Louvain-la-Neuve, Belgium; ${ }^{b} \mathrm{CHU}$ de Nantes, Department of Addictology and \\ Psychiatry, Clinical Investigation Unit 'Behavioral Addictions and Complex Mood Disorders', Nantes, France; \\ 'EA 4275 SPHERE 'MethodS for Patients-centered outcomes and HEalth Research', University of Nantes, \\ Nantes, France; ${ }^{\mathrm{P}}$ sychology and Neuroscience of Cognition Research Unit, University of Liège, Liège, Belgium; \\ eInternet and Gambling Disorders Clinic, Department of Adult Psychiatry, Cliniques Universitaires Saint-Luc, \\ Brussels, Belgium
}

\begin{abstract}
Previous research has identified specific gambling motives and linked them with both healthy and disordered gambling. The Gambling Motives Questionnaire (GMQ) is currently the most widely used measure for these motives. The present study aimed to offer a French validation of the latest version of this scale, the GMQ-Financial (GMQ-F), which measures four distinct motives (enhancement, social, coping, financial). The French GMQ-F was completed by 278 gamblers from the community and 22 treatment-seeking pathological gamblers, along with scales assessing gambling cognitions, impulsivity, disordered gambling symptoms and psychopathological symptoms. Confirmatory factor analysis supported the expected four-factor model. The GMQ-F subscales have good internal reliability. Validity of the GMQ-F is supported by specific correlations with the other constructs measured. Pathological gamblers differed from gamblers from the community on all but one (social) of the GMQ-F subscales. The French GMQ-F presents good psychometric properties and constitutes a reliable instrument for measuring gambling motives in research and clinical practice.
\end{abstract}

\section{ARTICLE HISTORY}

Received 5 July 2016

Accepted 17 November 2016

\section{KEYWORDS}

GMQ-F; gambling motives; problem gambling; gambling disorder; confirmatory factor analysis; gambling cognitions

Influential models clearly grant a central role to an individual's motives in substance-related and behavioural addictive habits (Jacobs, 1986; Orford, 2001). A large body of research has indeed emphasized the crucial influence of both positive (e.g. enhancement) and negative (e.g. coping) reinforcement-related motives in the onset, maintenance and relapse of addictive/excessive behaviours (Blaszczynski \& Nower, 2002; Hyman, Malenka, \& Nestler, 2006; Koob \& Le Moal, 2008) such as abuse of and dependence on alcohol (Lyvers, Hasking, Hani, Rhodes, \& Trew, 2010) and tobacco (Mathew et al., 2014), or disordered involvement in gambling

CONTACT Gaëtan Devos@gaetan.devos@uclouvain.be; Joël Billieux joel.billieux@uclouvain.be 
(Canale, Vieno, Griffiths, Rubaltelli, \& Santinello, 2015), video gaming (Billieux et al., 2013) and cybersex (Wéry \& Billieux, 2016). Beyond the global presence of enhancement and coping motives in all addictive or excessive behaviours, motives related to a specific activity have also been revealed (e.g. financial motive in gambling, Dechant, 2014; virtual immersion motive in video gaming, Yee, 2006; anonymous fantasizing motive in online sexual activities, Wéry \& Billieux, 2016).

Several models have been proposed to capture the main motives associated with gambling behaviours (Binde, 2013) and their potential role in the emergence of problematic gambling behaviours (Blaszczynski \& Nower, 2002; Milosevic \& Ledgerwood, 2010). Various scales have thus been developed to measure gambling motives, including the Gambling Motives Questionnaire (GMQ; Stewart \& Zack, 2008), the Gambling Motivation Scale (Chantal, Vallerand, \& Vallières, 1994) and the Reasons for Gambling Questionnaire (Canale, Santinello, \& Griffiths, 2015). Among these scales, the GMQ (Stewart \& Zack, 2008 ) is currently the most widely used in research on problematic and no problematic gambling. The GMQ is a self-reported questionnaire directly adapted from the Drinking Motives Questionnaire (Cooper, Russell, Skinner, \& Windle, 1992), which measures three types of gambling motives: (a) enhancement motives (i.e. gambling to search for stimulation, arousal or positive affect); (b) coping motives (i.e. gambling to relieve negative affect or boredom, or to escape real-life problems); and (c) social motives (i.e. gambling to enhance affiliation with others or to share moments with peers). On the whole, and in line with what has been found in substance abuse research, previous studies conducted with the GMQ identified that both enhancement and coping motives (but not social motives) are vulnerability factors for problem gambling (Lambe, Mackinnon, \& Stewart, 2015; Stewart $\&$ Zack, 2008). Moreover, although escapism motives appeared as the strongest predictor of problem gambling symptoms when gambling frequency was controlled for (Stewart \& Zack, 2008), both escapism and enhancement motives predicted gambling-related expenses beyond social motives (Lambe et al., 2015).

Models of gambling motives and empirical evidence also emphasized the existence of unique gambling motives that are not measured by the initial version of the GMQ. These motives also varied across game types (e.g. skill-based games such as poker are more frequently related to competition or intellectual challenge motives, whereas chance-based games are more frequently related to the dream of hitting the jackpot; see Binde, 2013). In particular, studies suggest that financial-related motives are common in gamblers. For example, a study by Neighbors, Lostutter, Cronce, and Larimer (2002) found that 'winning money' is one of the main reasons for gambling among college students. More recently, McGrath, Stewart, Klein, and Barrett (2010) investigated self-generated motives for gambling in community participants and found that $46 \%$ of the total motives that they reported involved financial considerations. Interestingly, adding a monetary-related item in the GMQ has also been shown to improve the psychometric properties of the instrument in a sample of moderately involved gamblers (Dechant \& Ellery, 2011).

As financial reasons appear ubiquitous among gamblers, claims have been made that the GMQ failed to measure this core component of gambling motives (Hodgins, 2008; McGrath et al., 2010). In response to this important limitation, Dechant (2014) developed a new version of the scale that incorporated a supplementary four-item financial subscale. Using confirmatory factor analytic techniques, Schellenberg, McGrath, and Dechant (2016) supported the expected four-factor structure of the GMQ-F. Initial results obtained with the GMQ-F showed that its financial subscale predicts gambling frequency but not problem gambling 
symptoms (Dechant, 2014; Schellenberg et al., 2016). However, the relationships between the financial subscale of the GMQ-F and other vulnerability factors for problem gambling (e.g. gambling-related cognitive distortions, impulsivity traits) remain to be explored.

The objective of the present study was to test whether the psychometric properties of the original English GMQ-F demonstrated by Schellenberg et al. (2016) can be replicated by using an adapted French version of the scale. Indeed, in view of the relevance of this scale for both research and clinical purposes, there is an urgent need to validate the GMQ-F for countries with French-speaking communities, representing more than 270 million people worldwide. To this end, a three-step procedure was followed. First, the factor structure of the French GMQ-F was tested by using a confirmatory factor analytic approach. Second, the relevance of the GMQ-F for problem gambling research was tested by exploring its links with (a) symptoms of disordered gambling; (b) signs of general psychopathology (anxiety, stress, depression); and (c) two types of psychological factors that have been repeatedly associated with disordered gambling, namely impulsivity traits (Billieux, Lagrange, et al., 2012; Blaszczynski \& Nower, 2002; Goudriaan, Oosterlaan, de Beurs, \& Van den Brink, 2004; Nower \& Blaszczynski, 2006) and gambling-related cognitions (Joukhador, Blaszczynski, \& Maccallum, 2004; Toneatto, 1999; Toneatto, Blitz-Miller, Calderwood, Dragonetti, \& Tsanos, 1997). Third, gambling motive profiles as measured by the GMQ-F were compared between occasional, regular and treatment-seeking pathological gamblers.

\section{Method}

\section{Participants and procedure}

An online survey was administered to 278 French-speaking gamblers from the community (132 men) aged from 18 to 78 years (Mean: $35.41 ; \mathrm{SD}=13.32$ ). The sample comprises $18 \%$ students, $72.7 \%$ active workers, $3.6 \%$ unemployed people and $5.7 \%$ retired people. The participants reported living in Belgium (58.3\%), France (37.1\%) or other countries (4.6\%). Participants were recruited through advertisements on social networks, research-related networks of the investigators, and public places in France and Switzerland. A small subgroup of participants $(n=28)$ completed the scale three times (with an interval of six months between each measure) to provide an estimation of test-retest stability. All participants gave their consent before starting the survey. Inclusion criteria were being at least 18 years old, being a fluent French speaker and reporting past-year gambling involvement. Anonymity and confidentiality were also guaranteed (no data regarding the identification of participants was collected, including their Internet Protocol address). The protocol was approved by the ethical committee of the Psychological Sciences Research Institute (IPSY) of the Université catholique de Louvain and by the local Research Ethics Committee of Nantes (GNEDS).

After having answered a demographic questionnaire and several questions assessing gambling involvement (type of gambling activities and gambling frequency), participants completed the French GMQ-F to measure gambling motives and the Problem Gambling Severity Index (PGSI; Ferris \& Wynne, 2001) to measure symptoms of disordered gambling. A subgroup of the sample $(n=217)$ also completed additional measures in random order: (a) the short version of the Depression Anxiety Stress Scale (DASS-21; Lovibond \& Lovibond, 1995); (b) the short UPPS-P Impulsive Behavior Scale (UPPS-P; Billieux et al., 2012); and (c) the Gambling-Related Cognitions Scale (GRCS; Raylu \& Oei, 2004). 
Table 1. Descriptive statistics and Cronbach's alphas of all scales for the two samples ( $n=278$ for the original sample and $n=22$ for the PG group) and statistical differences between community and treatment-seeking pathological gamblers.

\begin{tabular}{|c|c|c|c|c|c|c|c|}
\hline & \multicolumn{3}{|c|}{ Community gamblers } & \multicolumn{3}{|c|}{ PG } & \multirow{2}{*}{$\begin{array}{c}\text { Mann-Whitney test } \\
\text { (effect size) }\end{array}$} \\
\hline & $M$ & $S D$ & $a$ & $M$ & $S D$ & $a$ & \\
\hline GMQ-F Coping & 5.28 & 2.02 & .79 & 9.00 & 3.06 & .71 & $p<.001$ (1.59) \\
\hline GMQ-F Enhancement & 8.38 & 3.24 & .84 & 11.23 & 2.70 & .73 & $p<.001(0.87)$ \\
\hline GMQ-F Social & 4.01 & 1.52 & .74 & 4.32 & 1.64 & .67 & ns \\
\hline GMQ-F Financial & 9.53 & 3.42 & .81 & 11.55 & 2.60 & .73 & $p<.01(0.59)$ \\
\hline PGSI & 1.91 & 3.39 & .87 & 18.32 & 3.4 & .53 & $p<.001(2.81)$ \\
\hline GRCS-IB & 10.62 & 5.94 & .76 & 17.23 & 5.83 & .70 & $p<.001(1.06)$ \\
\hline GRCS-PC & 15.46 & 6.98 & .71 & 19.82 & 7.34 & .75 & $p<.01(0.61)$ \\
\hline GRCS-GE & 10.48 & 4.74 & .70 & 16.23 & 5.48 & .60 & $p<.001(1.13)$ \\
\hline GRCS-IS & 8.66 & 5.54 & .87 & 25 & 6.08 & .81 & $p<.001(2.23)$ \\
\hline GRCS-IC & 6.80 & 4.20 & .77 & 8.45 & 5.01 & .75 & ns \\
\hline UPPS-P-Negative Urgency & 9.36 & 2.78 & .82 & 10.96 & 2.55 & .70 & $p<.05(0.57)$ \\
\hline UPPS-P-Positive Urgency & 10.47 & 2.66 & .82 & 11.68 & 2.67 & .69 & ns \\
\hline UPPS-P-Premeditation & 7.23 & 2.36 & .85 & 8.36 & 2.38 & .79 & $p<.05(0.47)$ \\
\hline UPPS-P-Perseverance & 7.21 & 2.45 & .85 & 8.09 & 2.41 & .74 & ns \\
\hline UPPS-P-Sensation & 10.32 & 2.86 & .85 & 8.32 & 2.62 & .82 & $p<.01(0.69)$ \\
\hline DASS-21-Depression & 4.23 & 4.62 & .90 & & & & \\
\hline DASS-21-Anxiety & 2.97 & 3.30 & .78 & & & & \\
\hline DASS-21-Stress & 6.13 & 4.78 & .87 & & & & \\
\hline
\end{tabular}

Note: $P G=$ Treatment-seeking pathological gamblers; GMQ-F = Gambling Motives Questionnaire-Financial; UPPS-P = UPPS-P Impulsive Behavior Scale; DASS = Depression Anxiety and Stress Scale; PGSI = Pathological Gambling Severe Index; GRCSIB = Gambling-Related Cognitions Scale-Interpretative Bias; GRCS-PC = Gambling-Related Cognitions Scale-Predictive Control; GRCS-GE = Gambling-Related Cognitions Scale-Gambling Expectancies; GRCS-IS = Gambling-Related Cognitions Scale-Perceived Inability to Stop Gambling; GRCS-IC = Gambling-Related Cognitions Scale-Illusion of Control.

An additional group of 22 treatment-seeking patients (18 men), aged between 23 and 67 (Mean = 41.27; SD = 12.74), was recruited at the Department of Addictology and Psychiatry of the Nantes University Hospital (France). All patients were enrolled in a special treatment programme targeting pathological gambling, and completed a paper-and-pencil version of the research protocol at the initial stage of their treatment. All patients fulfilled the DSMIV-TR criteria for pathological gambling.

Descriptive statistics, the internal consistency coefficient (Cronbach's $\alpha$ ) and comparisons between healthy gamblers and pathological gamblers for all questionnaires are reported in Table 1.

\section{Measures}

\section{Gambling frequency and types of gambling activities}

A questionnaire evaluating gaming type and frequency was used. First, we asked participants if they played, during the last year, seven of the most frequent gambling activities: lottery, poker, online poker, scratch cards, betting, slot machine and others. Second, with a positive answer to the first question, we asked participants how often they played this gambling activity ( $1=$ a few times in the last year, $2=$ once a month, $3=$ a few times per month, 4 = once a week, 5 = a few times a week, and 6 = every day or so).

\section{The Gambling Motives Questionnaire-Financial (GMQ-F)}

The French GMQ-F consisted of 16 items translated from the original English version (Dechant, 2014). The French version of the questionnaire was developed as follows: 
(a) two authors (GCB and MGB) of this study translated the 16 items of the original GMQ-F; (b) an English-French bilingual translator translated the French version back into English; and (c) all discrepancies identified between the original GMQ-F and the back-translation were discussed until a satisfactory solution was found. The scale measures four main gambling motives: coping (e.g. 'because it helps when you are feeling nervous or depressed'), enhancement (e.g. 'because it's exciting'), social ('because it makes a social gathering more enjoyable') and financial (e.g. 'because you enjoy thinking about what you would do if you win a jackpot'). Items were rated on a 4-point Likert scale ranging from 1 (never or almost never) to 4 (almost always or always).

\section{The short version of the Depression, Anxiety and Stress Scale (DASS-21)}

The DASS-21 was translated into French by Lovibond and Lovibond (1995), contains three seven-item subscales, measuring the severity and frequency of symptoms of depression ('I found it difficult to work up the initiative to do things'), anxiety ('I was worried about situations in which I might panic and make a fool of myself') and stress ('I found myself getting agitated'). Items were scored on a 4-point Likert scale, ranging from 0 (did not apply to me at all) to 3 (applied to me very much). The total sum for DASS-21 was derived by summing the total scores of all individual items.

\section{Problem Gambling Severity Index (PGSI)}

The French translation of the PGSI was given as a module within the Canadian Problem Gambling Inventory (Ferris \& Wynne, 2001). These authors translated and validated the French version. PGSI items were answered on a 4 -point Likert scale $(0=$ never, $1=$ sometimes, 2 = most of the time, 3 = always or almost always). The total score, calculated by summing items, ranged from 0 to 27. In the current study, the PGSI score was considered a dimensional measure of problem gambling symptoms.

\section{UPPS-P Impulsive Behavior Scale (UPPS-P)}

The UPPS-P (Billieux et al., 2012) is a 20-item scale created and validated in French which assesses 5 dimensions of impulsivity: negative urgency (i.e. the tendency to act rashly when experiencing intense negative affect), positive urgency (i.e. the tendency to act rashly when experiencing intense positive affect), lack of premeditation (i.e. difficulty in taking into account the consequences of an act before engaging in that act), lack of perseverance (i.e. difficulty in remaining focused on a task that may be boring and/or difficult) and sensation seeking (i.e. the tendency to enjoy and pursue activities that are stimulating or exciting and openness to trying new and unconventional experiences). Items were answered on a 4-point Likert scale ranging from 1 (I strongly agree) to 4 (I strongly disagree).

\section{Gambling-Related Cognitions Scale (GRCS)}

The French version of the GRCS (Grall-Bronnec et al., 2012), adapted from Raylu and Oei (2004), is a 23-item scale assessing a variety of gambling-related cognitions. The GRCS measures five facets: interpretative bias (cognitions related to reframing of gambling outcomes; e.g. 'Relating my winnings to my skill and ability makes me continue gambling'); illusion of control (cognitions related to the ability to control gambling outcomes; e.g. 'Specific numbers and colors can help increase my chances of winning'); predictive control (cognitions related to the ability to predict gambling outcomes; e.g. 'I have some control 
over predicting my gambling wins'); gambling expectancies (positive reinforcement value, e.g. excitement, or negative reinforcement value, e.g. relief of negative mood or boredom; e.g. 'Gambling makes the future brighter'); and perceived inability to stop gambling (beliefs about the ability to stop or control gambling, e.g. 'It is difficult to stop gambling as I am so out of control'). Items were answered on a 7-point Likert scale ranging from 1 (strongly disagree) to 7 (strongly agree).

\section{Statistical analysis}

To determine the factor structure of the GMQ-F, we analysed the covariance matrix with a confirmatory factor analysis (CFA) computed with LISREL 8.8 (Jöreskog \& Sörbom, 1996). Considering that the multi-normality condition was not respected, all models were computed by using maximum likelihood estimation with robust standard errors. We used CFA instead of exploratory factor analysis because the former allows testing specific a priori hypotheses regarding the factorial structure of the scale, which is particularly suited for adaptation of scales that have received prior validations. Two models were computed and compared: a one-factor model and a four-factor model. In accordance with Dechant (2014), the four extracted factors were coping, enhancement, social and financial. Goodness of fit was tested with the $\chi^{2}$ test (a non-significant value corresponds to an acceptable fit). However, $\chi^{2}$ is known to increase with sample size, and Byrne (1994) has noticed that it is unusual to obtain a non-significant $\chi^{2}$ when performing CFA on self-report questionnaires. We therefore completed the $\chi^{2}$ by examining other indices that depend on conventional cut-offs. The following indices were consequently reported: root mean square error of approximation (RMSEA), comparative fit index (CFI), adjusted goodness of fit index (AGFI), normed fit index (NFI) and non-normed fit index (NNFI). An RMSEA of <.08, a CFI of $>.95$, an AGFI of $>.85$, an NFI of $>.90$ and an NNFI of $>.95$ are generally interpreted as acceptable fits. Because Item 9 was found to have a negative impact on the internal consistency of the social factor in previous validations of the GMQ and GMQ-F (see Dechant, 2014; Lambe et al., 2015), the same two models were also computed without this item.

Moreover, the expected cross-validation index (ECVI) was used to compare the different models. The ECVI measures whether a model is likely to cross-validate across samples of the same size from the same population. The smaller the ECVI, the greater the potential the model has for replication (Diamantopoulos \& Siguaw, 2008).

Spearman correlations (with 5\% significance criterion) were used to test the relations between the variables included in the study. Pearson correlations were also used to explore test-retest stability of the French GMQ-F. Variables were recoded to consider gender effect (female $=1$; male $=0$ ) and gambling types (involvement in a certain type of gambling activity last year $=1$; no involvement $=0$ ). The sample of gamblers from the community was divided into two groups on the basis of the frequency of play to evaluate differences among the subscales of the GMQ-F through analyses of variance. The first group was composed of gamblers who played at least once a week, called 'Regular Gamblers', and the second group was composed of gamblers who played less than once a week, called 'Occasional Gamblers'. Moreover, these groups were compared to the pathological gamblers in treatment. We used the Benjamini-Hochberg procedure (Benjamini \& Hochberg, 1995) to hold the false discovery rate at $5 \%$ for the multiple analyses conducted. 


\section{Results}

\section{Variation in gambling behaviour}

Gambling frequencies were as follow: less than monthly $(n=25,9 \%)$, at least monthly $(n=165,59.4 \%)$, at least once a week $(n=33,11.8 \%)$, more than once a week $(n=55$, $19.8 \%)$. Represented gambling types were lotteries (68.8\%), scratch cards (58.8\%), table poker $(26.5 \%)$, slot machines (14\%), online poker (16.1\%), sports betting (14\%) and others (9\%). PGSI scores ranged from 0 to $21(M=1.91, \mathrm{SD}=3.39)$. According to the proposed cut-off for the PGSI (Ferris \& Wynne, 2001), 50.69\% of the sample scored 0 (no gambling risk), $26.26 \%$ scored 1 or 2 (low risk to have problems with gambling), $16.12 \%$ scored 3 to 7 (moderate risk to have problems with gambling) and $6.91 \%$ scored 8 or more (high risk to have problems with gambling or loss of control).

\section{Structural validity}

The 16 items of the GMQ-F were submitted to a confirmatory analysis with no missing value observed. Four models were computed. In each model, the maximum modification indices in the $\Theta-\Delta$ matrix (covariance between errors on observed variables) were found between Items 1 and 5 and Items 14 and 15. We allowed the two pairs of errors to covary, as Items 1 and 5 both refer to social relations with friends and Items 14 and 15 both refer to mood regulation.

In the one-factor model (Model 1), the $\chi^{2}$ statistic was significant, $\chi^{2}(102)=949.695$, $p<.001$. The RMSEA (.159), CFI (.778), AGFI (.553), NFI (.755) and NNFI (.739) clearly indicated that this one-factor model has a poor fit.

To define a model with four dimensions (Model 2), we allowed the four latent variables to correlate. Causality relations were calculated for the following latent variables: (a) coping (Items 2, 6, 10, 14); (b) enhancement (Items 3, 7, 11, 15); (c) social (Items 1, 5, 9, 13); and (d) financial (Items $4,8,12,16)$. The $\chi^{2}$ statistic was significant, $\chi^{2}(96)=315.794(p<.001)$. The RMSEA (.0780), CFI (.949), AGFI (.820), NFI (.922) and NNFI (.937) showed that this four-factor model has a reasonable fit.

The 2 same models were also computed with only 15 items (i.e. after having removed Item 9). In the one-factor model (Model $1^{\prime}$ ), the $\chi^{2}$ statistic was significant, $\chi^{2}(88)=918.064$ $(p<.001)$. We allowed the two pairs of errors to covary. The RMSEA (.167), CFI (.785), AGFI (.534), NFI (.765) and NNFI (.744) showed that this one-factor model has a poor fit.

In the four-factor model (Model 2'; see Figure 1), the $\chi^{2}$ statistic was significant, $\chi^{2}(82)$ $=282.762(p<.001)$. The RMSEA (.0791), CFI (.955), AGFI (.824), NFI (.932) and NNFI (.943) showed that this four-factor model has a reasonable fit. The ECVI comparison index indicated that Model 2' $(\mathrm{ECVI}=1.083)$ has a better fit than Model $1(\mathrm{ECVI}=3.180)$, Model $1^{\prime}(\mathrm{ECVI}=3.003)$ and Model $2(\mathrm{ECVI}=1.220)$.

\section{Descriptive statistics, internal consistency and reliability, test-retest stability and correlation between all facets of the GMQ-F}

Table 2 displays descriptive statistics, scale score reliability indices and test-retest stability of the French version of the GMQ-F (total score and four facets). All Cronbach's alpha coefficients were higher than .75 , thereby indicating good scale and facet score reliabilities 


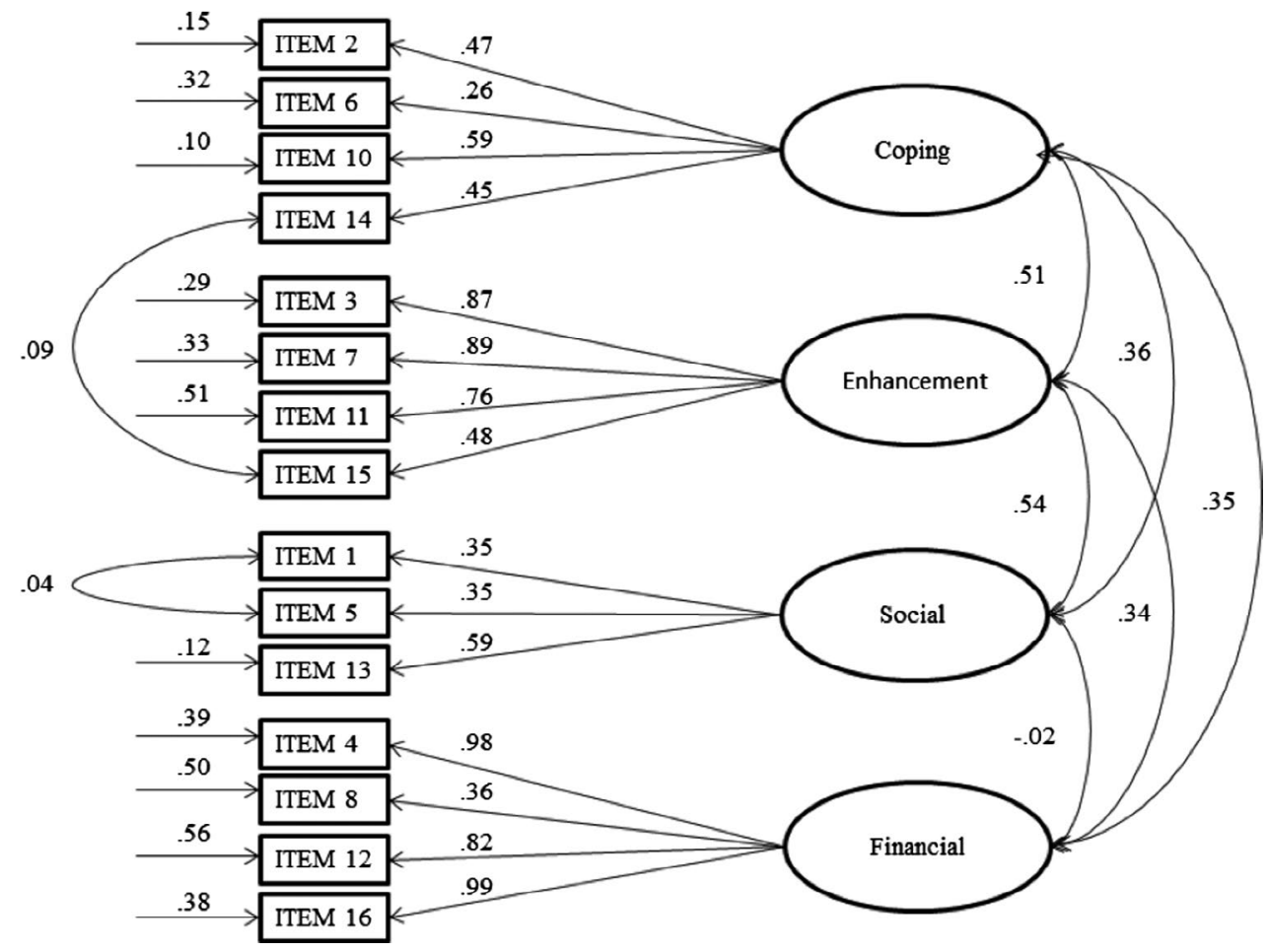

Figure 1. A four-factor model (Model 2') in which all latent variables are represented by ovals and all manifest variables are represented by rectangles. Single-headed arrows represent error variance and factor loading; double-headed arrows represent the correlation between the latent variable.

Table 2. Descriptive statistics, test-retest stability and intercorrelations among the GMQ-F dimensions $(N=278)$.

\begin{tabular}{|c|c|c|c|c|c|c|c|c|c|}
\hline & \multicolumn{9}{|c|}{ Factor intercorrelation } \\
\hline & $M$ & $S D$ & a & $\begin{array}{c}\text { Retest } \\
\text { (6 months) }\end{array}$ & $\begin{array}{l}\text { Retest } \\
\text { (1 year) }\end{array}$ & 1 & 2 & 3 & 4 \\
\hline $1 \mathrm{GMQ}-\mathrm{F}$ total & 26.15 & 10.63 & .83 & $.683^{* * *}$ & $.738^{* * *}$ & - & - & - & - \\
\hline $\begin{array}{l}2 \text { GMQ-F - } \\
\text { Coping }\end{array}$ & 5.28 & 2.02 & .79 & $.437^{*}$ & $.525^{* *}$ & $0.60^{* * *}$ & - & - & - \\
\hline $\begin{array}{c}3 \mathrm{GMQ}-\mathrm{F} \text { - En- } \\
\text { hancement }\end{array}$ & 8.38 & 3.24 & .84 & $.772^{* * *}$ & $.681^{* * *}$ & $0.68^{* * *}$ & $0.52^{* * * *}$ & - & - \\
\hline $\begin{array}{l}4 \text { GMQ-F - } \\
\text { Social }\end{array}$ & 4.01 & 1.52 & .74 & $.642^{* * *}$ & $.630 * * *$ & $0.60^{* * *}$ & $0.37^{* * *}$ & $0.77^{* * *}$ & - \\
\hline $\begin{array}{l}5 \text { GMQ-F - } \\
\text { Financial }\end{array}$ & 9.53 & 3.42 & .81 & $.700^{* * *}$ & $.640 * * *$ & $0.56^{* * *}$ & $0.35^{* *}$ & $0.30 * * *$ & $0.15^{*}$ \\
\hline
\end{tabular}

Note: GMQ-F = Gambling Motives Questionnaire-Financial.

${ }^{*} p<.05 ;{ }^{* *} p<.01 ;{ }^{* * *} p<.001$.

(Nunnally, 1978) except for the social facet (.74), which has acceptable reliability. As expected, all facets with a good internal consistency are significantly intercorrelated.

\section{Correlation between GMQ-F, all questionnaires, sex and age}

As presented in Table 3, positive relations are shown between the GMQ-F and the other constructs linked to gambling. The main correlations are presented here according to their 
Table 3. Spearman correlations between all GMQ-F dimensions and age $(N=278)$, gender $(N=278)$, PGSI $(N=278)$, GRCS, UPPS, DASS $(n=217)$ and prevalence of gambling type played $(N=278)$.

\begin{tabular}{lcccc}
\hline & GMQ-F-Coping & GMQ-F-Enhancement & GMQ-F Social & GMQ-F-Financial \\
\hline Age & $-0.15^{* *}$ & $-0.30^{* * *}$ & $-0.22^{* * *}$ & -0.08 \\
Sex & 0.01 & -0.11 & -0.10 & $0.15^{*}$ \\
PGSI & $0.46^{* * *}$ & $0.55^{* * *}$ & $0.27^{* * *}$ & $0.16^{*}$ \\
GRCS-IB & $0.45^{* * *}$ & $0.62^{* * *}$ & $0.39^{* * *}$ & $0.29^{* * *}$ \\
GRCS-PC & $0.51^{* * *}$ & $0.53^{* * * *}$ & $0.37^{* * *}$ & $0.37^{* * * *}$ \\
GRCS-GE & $0.53^{* * *}$ & $0.55^{* * *}$ & $0.28^{* * *}$ & $0.31^{* * *}$ \\
GRCS-IS & $0.50^{* * *}$ & $0.49^{* * *}$ & $0.29^{* * *}$ & $0.24^{* * *}$ \\
GRCS-IC & $0.38^{* * *}$ & $0.32^{* * *}$ & $0.21^{* *}$ & $0.25^{* * *}$ \\
Negative urgency & $0.13^{* *}$ & -0.02 & 0.02 & $0.18^{* * *}$ \\
Positive urgency & $0.17^{* *}$ & $0.22^{* *}$ & $0.15^{*}$ & $0.23^{* * *}$ \\
Lack of premeditation & $0.16^{*}$ & $0.16^{*}$ & 0.03 & 0.06 \\
Lack of perseverance & 0.09 & $0.14^{*}$ & 0.06 & -0.04 \\
Sensation seeking & 0.11 & $0.27^{* * *}$ & $0.19^{*}$ & 0.06 \\
DASS-21 - Depression & $0.25^{* * *}$ & -0.03 & 0.06 & $0.25^{* * *}$ \\
DASS-21 - Anxiety & $0.28^{* * *}$ & 0.10 & $0.19^{* *}$ & $0.20^{* *}$ \\
DASS-21 - Stress & $0.31^{* * *}$ & $0.15^{*}$ & $0.17^{*}$ & $0.28^{* * *}$ \\
Playing lottery & 0.07 & -0.02 & -0.02 & $0.38^{* * *}$ \\
Playing poker & $0.19^{* *}$ & $0.40^{* * *}$ & $0.36^{* * *}$ & -0.11 \\
Playing scratch cards & $0.14^{* *}$ & $0.18^{* *}$ & 0.03 & $0.29^{* * *}$ \\
Betting & $0.15^{*}$ & $0.24^{* * *}$ & $0.16^{* *}$ & 0.09 \\
Playing slot machine & $0.17^{*}$ & $0.21^{* * *}$ & $0.13^{*}$ & -0.06 \\
\hline
\end{tabular}

Note: PGSI = Pathological Gambling Severe Index; GRCS-IB = Gambling-Related Cognitions Scale-Interpretative Bias; GRCSPC = Gambling-Related Cognitions Scale-Predictive Control; GRCS-GE = Gambling-Related Cognitions Scale-Gambling Expectancies; GRCS-IS = Gambling-Related Cognitions Scale-Perceived Inability to Stop gambling; GRCS-IC = Gambling-Related Cognitions Scale-Illusion of Control; DASS = Depression Anxiety and Stress Scale. ${ }^{*} p<.05 ;{ }^{* *} p<.01 ;{ }^{* * *} p<.001$.

size effects on the basis of Cohen's terminology (Cohen, 1988) ('small' effect size, $r=.10$; 'medium' effect size, $r=.30$; and 'large' effect size, $r=.50)$.

Correlations appeared between all subscales of the GMQ-F and symptoms of disordered gambling (PGSI), with a large effect size for the enhancement subscale, a medium effect size for the coping subscale and a small effect size for the social and financial subscales.

The subscales of the GMQ-F are correlated with almost all types of gambling-related cognitions assessed with the GRCS. However, when only considering the correlations with a large effect size, the following links can be emphasized: coping is strongly related to predictive control, gambling expectancies and inability to stop gambling; and enhancement is strongly related to interpretative bias, predictive control and gambling expectancies.

Despite the small effect sizes, relations exist between the GMQ-F and impulsivity (UPPS-P). First, enhancement is related to all impulsivity facets except negative urgency. Second, coping is related to negative urgency, positive urgency and lack of premeditation. Third, social is related to positive urgency and sensation seeking. Finally, financial is related to positive urgency and negative urgency.

Relationships also appeared between the GMQ-F subscales and psychopathological symptoms. Medium effect size relations were observed between coping and stress, and several small effect size relations were also seen. Coping is related to depression and anxiety. Enhancement is related to stress. Social is related to anxiety and stress. Finally, financial is related to depression, anxiety and stress.

Associations between gambling preferences (i.e. type of gambling activities practised) and GMQ-F subscales were found. First, small effect size relationships were found between coping and the following types of gambling activities: poker, scratch cards, slot machines 

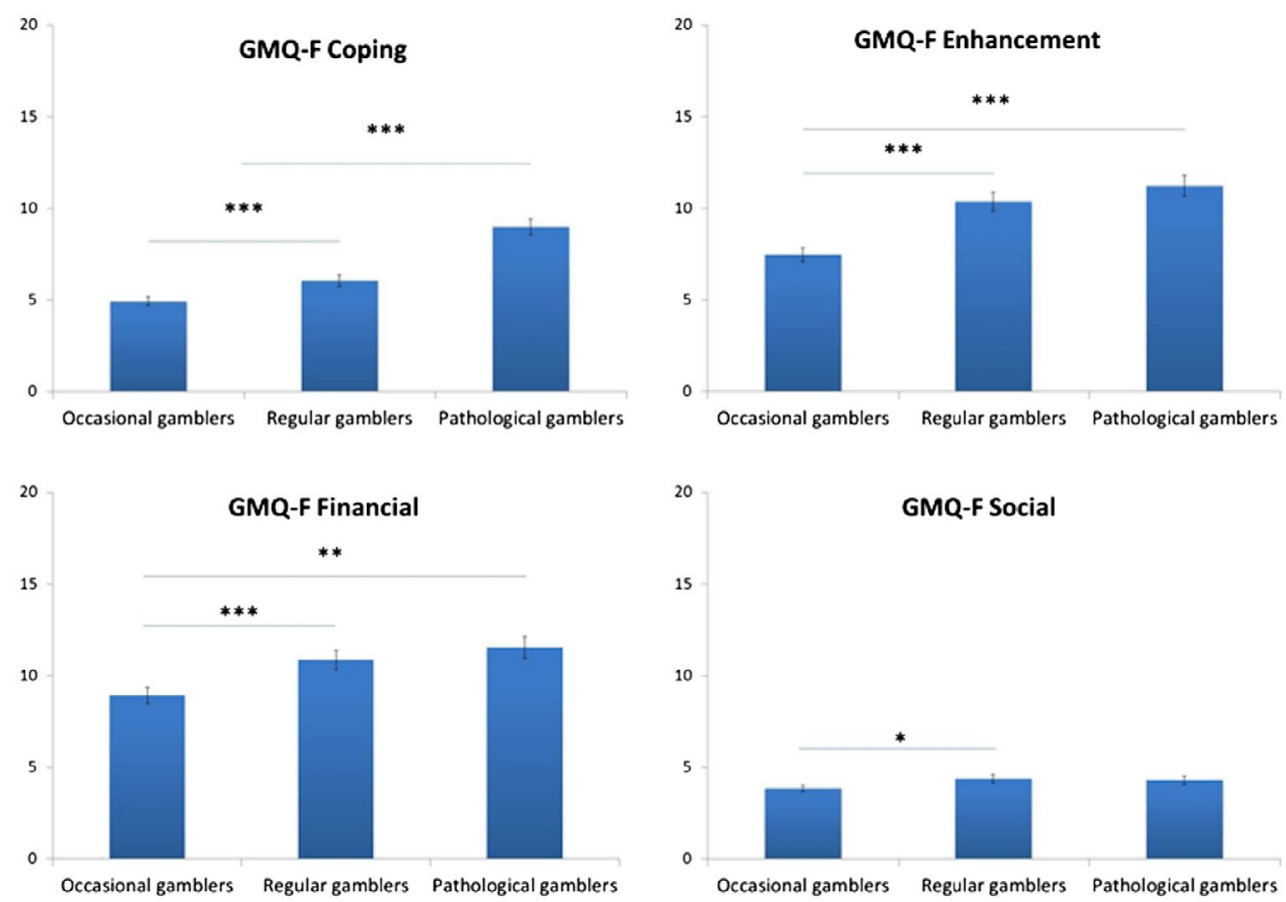

Figure 2. Comparisons on the four subscales of the Gambling Motives Questionnaire-Financial (GMQ-F) between three groups of gamblers: (occasional, $n=190$; regular, $n=88$; and pathological in treatment $(\mathrm{n}=22)$. Note: ${ }^{*} \mathrm{p}<.05 .{ }^{*}, \mathrm{p}<.01 .{ }^{* *}, \mathrm{p}<.001^{* * *}$.

and bets. Second, a moderate relationship was found between enhancement and being a poker player, whereas small effect size correlations were found between this motive and the following gambling activities: scratch cards, slot machines and bets. Third, a moderate relationship appeared between social and being a poker player, whereas small effect size relations took place between this motive and slot machines and bets. Finally, a moderate correlation was observed between the financial motive and being a lottery player, whereas a small amplitude correlation also took place between this motive and playing scratch cards.

\section{Group comparisons}

Group comparisons showed significant group (occasional, regular and pathological gamblers $)$ differences on the Coping $(\mathrm{F}(2,298)=42.327, \mathrm{p}<.001)$, Enhancement $(\mathrm{F}(2,298)=$ 39.303, $\mathrm{p}<.001)$, Social $(\mathrm{F}(2,298)=4.271, \mathrm{p}<.05)$ and Financial $(\mathrm{F}(2,298)=14.431, \mathrm{p}<.001)$ subscales. Figure 2 depicts post hoc comparisons.

\section{Discussion}

In the present study, we examined the psychometric properties of a French version of the GMQ-F, and showed (by means of a CFA identifying an ideal four-facet model) the same structure and psychometric properties as those initially found in the English version (Dechant, 2014). In line with previous studies (Dechant, 2014; Lambe et al., 2015), 
a 15-item model was chosen, excluding a problematic item (Item 9, related to the social subscale). Internal consistency of the various scales indicated good scale reliability. The test-retest stability of the subscales is high, excepted for the coping motive, where the correlations are lower ${ }^{1}$. Complementary analyses identified relations between GMQ-F and related constructs (gambling frequency and preferences, disordered gambling symptoms, psychopathological symptoms, gambling-related cognitions, impulsivity). On the whole, this study demonstrated that the French version of the GMQ-F is characterized by good psychometric properties.

Crucially, the current study confirmed that the theory-based factorial structure of the GMQ-F allows us to disentangle the effects of various motives on gambling preferences and problematic gambling. First, we emphasized that regular gamblers, in comparison with occasional gamblers, presented more pronounced gambling motives (especially coping, enhancement and financial motives). Second, gambling motives was found to influence the preferential game type (see Table 3). In particular, it appears that (a) the status of the lottery player is related only to the financial motive; (b) poker is the gambling activity presenting the strongest links with enhancement and social motives; and (c) with the notable exception of the lottery, all gambling types are to some degree correlated with both coping and enhancement motives. Third, our study confirms and extends current knowledge about the relations between gambling motives and disordered gambling, by combining dimensional (PGSI being used as a continuous index of problem gambling in community gamblers) and categorical (community gamblers being compared with treatment-seeking pathological gamblers) approaches. Centrally, the joint use of dimensional and categorical approaches confirms that coping and enhancement motives are, among GMQ-F variables, the best indicators of disordered gambling, which is consistent with previous results (Lambe et al., 2015; Stewart \& Zack, 2008) and with dominant models of gambling addiction (Blaszczynski \& Nower, 2002; Jacobs, 1986). In contrast, the links between both social and financial and disordered gambling symptoms (PGSI) are of weaker amplitude $(r<.30)$, and the social motive was the only GMQ-F subscale that failed to distinguish community gamblers from pathological gamblers. The fact that the social motive has a small predictive value for problematic behaviours is consistent with previous studies from both the gambling and alcohol research fields (Cooper et al., 1992; Lambe et al., 2015; Schellenberg et al., 2016). The relationships identified between gambling motives and psychopathological symptoms are consistent with previous evidence suggesting that disordered gambling frequently occurs jointly with emotional disorders (Petry, Stinson, \& Grant, 2005) and that gambling behaviours can be used as a coping strategy to face negative affect or stress (Lightsey \& Hulsey, 2002; Wood \& Griffiths, 2007).

This study constitutes, to our knowledge, one of the first explorations of the links between gambling-related cognitions and gambling motives. Although the relations observed between gambling expectancies and both coping and enhancement motives were expected and can be linked to the emotion regulation function of gambling described earlier (see e.g. Wu, Tao, Tong, and Cheung 2012), other observed relations are innovative. Coping was the motive presenting the strongest links with perceived inability to stop gambling, a dysfunctional metacognitive belief that has been related to persistent playing despite losses in a laboratory task modelling slot machine gambling (Billieux, Van der Linden, Khazaal, Zullino, \& Clark, 2012). Second, relations of relatively high amplitudes were found between coping and enhancement motives and two types of gambling-related cognitive distortions, 
namely predictive control and interpretative bias. As no causality can be derived from the current correlational approach, further experimental studies are required to test whether the perspective of positive or negative reinforcement associated with gambling fosters or is conversely fostered by 'irrational' beliefs involving the influence of personal skills in gambling outcomes.

Despite their smaller amplitude, specific associations that occurred between gambling motives and impulsivity facets should be highlighted. In particular, it appears that positive urgency is related to all types of gambling motives, which can be related to the results of previous studies that emphasized the important predictive value of this impulsivity facet on the onset of disordered gambling (Blain, Richard Gill, \& Teese, 2015; Cyders \& Smith, 2008). The differential associations we found here between gambling motives and impulsivity facets globally match those obtained in an earlier study by Canale et al. (2015) using similar instruments (GMQ and UPPS-P). Although beyond the scope of the current article, these associations call for further explorations of the complex interactions or mediation effects between gambling motives and impulsivity, which could constitute pathways to problem gambling, in line with data obtained in alcohol abuse and dependence (Adams, Kaiser, Lynam, Charnigo, \& Milich, 2012; Curcio \& George, 2011).

Two limitations of the present study have to be acknowledged. First, our results relied on a small sample. Nonetheless, the sample size used is adapted to the analyses conducted and participants were all gamblers from the community (e.g. rather than undergraduate students) or pathological gamblers seeking treatment. Second, gamblers from the community were recruited through a self-selected online survey, which might have affected the representativeness of the sample; for example, by including a potentially higher proportion of individuals who perceived their gambling behaviours as disturbing or problematic (Khazaal et al., 2014).

The French GMQ-F presents good psychometric properties and can be considered a reliable instrument to measure gambling motives in research and clinical practice. Despite the above-mentioned limitations, the present study, by offering a sound validation of an important questionnaire in the gambling field, is a significant step towards better identification of gambling motives across the various categories of gamblers.

\section{Note}

1. A potential explanation to the lower test-retest correlation observed for the coping subscale is that the situations in which gambling is displayed as a coping strategy can be transient (e.g. a temporary dysphoric state that can be relied to a specific negative life event).

\section{Conflicts of interest}

\section{Funding sources}

This study was supported by a research grant obtained from the Belgium National Lottery. Pierre Maurage (research associate) is funded by the Belgian Fund for Scientific Research (FRS-FNRS, Belgium). Marie Grall-Bronnec and Gaëlle Challet-Bouju declare that the University Hospital of Nantes has received funding from the gambling industry (FDJ and 
PMU) in the form of a sponsorship that supports the gambling section of the Clinical Investigation Unit 'Behavioral Addictions and Complex Mood Disorders' The various entities mentioned did not exert any editorial influence over this article.

\section{Competing interests}

The authors declare no potential competing interests with respect to the research, authorship and/or publication of this article.

\section{Constraints on publishing}

No constraints on publishing were reported by the authors.

\section{ORCID}

Gaëlle Challet-Bouju (D) http://orcid.org/0000-0002-2238-8005

\section{References}

Adams, Z. W., Kaiser, A. J., Lynam, D. R., Charnigo, R. J., \& Milich, R. (2012). Drinking motives as mediators of the impulsivity-substance use relation: Pathways for negative urgency, lack of premeditation, and sensation seeking. Addictive Behaviors, 37, 848-855. http://doi.org/10.1016/ j.addbeh.2012.03.016

Benjamini, Y., \& Hochberg, Y. (1995). Controlling the false discovery rate: A practical and powerful approach to multiple testing. Journal of the Royal Statistical Society. Series B (Methodological), 57, 289-300.

Billieux, J., Lagrange, G., Van der Linden, M., Lançon, C., Adida, M., \& Jeanningros, R. (2012). Investigation of impulsivity in a sample of treatment-seeking pathological gamblers: A multidimensional perspective. Psychiatry Research, 198, 291-296.

Billieux, J., Rochat, L., Ceschi, G., Carré, A., Offerlin-Meyer, I., Defeldre, A.-C., ... Van der Linden, M. (2012). Validation of a short French version of the UPPS-P impulsive behavior scale. Comprehensive Psychiatry, 53, 609-615. http://doi.org/10.1016/j.comppsych.2011.09.001

Billieux, J., Van der Linden, M., Achab, S., Khazaal, Y., Paraskevopoulos, L., Zullino, D., \& Thorens, G. (2013). Why do you play world of warcraft? An in-depth exploration of self-reported motivations to play online and in-game behaviours in the virtual world of Azeroth. Computers in Human Behavior, 29, 103-109. http://doi.org/10.1016/j.chb.2012.07.021

Billieux, J., Van der Linden, M., Khazaal, Y., Zullino, D., \& Clark, L. (2012). Trait gambling cognitions predict near-miss experiences and persistence in laboratory slot machine gambling. British Journal of Psychology, 103, 412-427. http://doi.org/10.1111/j.2044-8295.2011.02083.x

Binde, P. (2013). Why people gamble: A model with five motivational dimensions. International Gambling Studies, 13, 81-97.

Blain, B., Richard Gill, P., \& Teese, R. (2015). Predicting problem gambling in Australian adults using a multifaceted model of impulsivity. International Gambling Studies, 15, 239-255. http://doi.org/ $10.1080 / 14459795.2015 .1029960$

Blaszczynski, A., \& Nower, L. (2002). A pathways model of problem and pathological gambling. Addiction, 97, 487-499. http://doi.org/10.1046/j.1360-0443.2002.00015.x

Byrne, B. M. (1994). Structural equation modeling with EQS and EQS/Windows. Thousand Oaks, CA: Sage.

Canale, N., Santinello, M., \& Griffiths, M. D. (2015). Validation of the reasons for Gambling Questionnaire (RGQ) in a british population survey. Addictive Behaviors, 45, 276-280. http://doi. org/10.1016/j.addbeh.2015.01.035 
Canale, N., Vieno, A., Griffiths, M. D., Rubaltelli, E., \& Santinello, M. (2015). How do impulsivity traits influence problem gambling through gambling motives? The role of perceived gambling risk/benefits. Psychology of Addictive Behaviors, 29, 813-823. http://doi.org/10.1037/adb0000060

Chantal, Y., Vallerand, R. J., \& Vallières, E. F. (1994). Assessing motivation to gamble: On the development and validation of the gambling motivation scale. Society and Leisure, 17, 189-212.

Cohen, J. (1988). Statistical power analysis for the behavioral sciences. Hillsdale, NJ: Erlbaum, Lawrence, Associates.

Cooper, M. L., Russell, M., Skinner, J. B., \& Windle, M. (1992). Development and validation of a three-dimensional measure of drinking motives. Psychological Assessment, 4, 123-132.

Curcio, A. L., \& George, A. M. (2011). Selected impulsivity facets with alcohol use/problems: The mediating role of drinking motives. Addictive Behaviors, 36, 959-964. http://doi.org/10.1016/ j.addbeh.2011.05.007

Cyders, M. A., \& Smith, G. T. (2008). Clarifying the role of personality dispositions in risk for increased gambling behavior. Personality and Individual Differences, 45, 503-508. http://doi. org/10.1016/j.paid.2008.06.002

Dechant, K. (2014). Show me the money: Incorporating financial motives into the gambling motives questionnaire. Journal of Gambling Studies, 30, 949-965. http://doi.org/10.1007/s10899-013-9386-5

Dechant, K., \& Ellery, M. (2011). The effect of including a monetary motive item on the gambling motives questionnaire in a sample of moderate gamblers. Journal of Gambling Studies, 27, 331-344. http://doi.org/10.1007/s10899-010-9197-x

Diamantopoulos, A., \& Siguaw, J. (2008). Introducing LISREL: A guide for the uninitiated. London, UK: Sage.

Ferris, J., \& Wynne, H. (2001). The Canadian gambling index final report. Ottawa: ON: Ottawa Canadian Centre on Substance Abuse.

Goudriaan, A. E., Oosterlaan, J., de Beurs, E., \& Van den Brink, W. (2004). Pathological gambling: a comprehensive review of biobehavioral findings. Neuroscience \& Biobehavioral Reviews, 28, $123-141$.

Grall-Bronnec, M., Bouju, G., Sébille-Rivain, V., Gorwood, P., Boutin, C., Vénisse, J.-L., \& Hardouin, J.-B. (2012). A French adaptation of the Gambling-Related Cognitions Scale (GRCS): A useful tool for assessment of irrational thoughts among gamblers. Journal of Gambling Issues, 27, 1-21. http://doi.org/10.4309/jgi.2012.27.9

Hodgins, D. C. (2008). [Commentary] What we see depends mainly on what we Look For (John Lubbock, British Anthropologist, 1834-1913). Addiction, 103, 1118-1119. http://doi.org/10.1111/ j.1360-0443.2008.02282.x

Hyman, S. E., Malenka, R. C., \& Nestler, E. J. (2006). Neural mechanisms of addiction: The role of reward-related learning and memory. Annual Review of Neuroscience, 29, 565-598.

Jacobs, D. F. (1986). A general theory of addictions: A new theoretical model. Journal of Gambling Behavior, 2, 15-31.

Joukhador, J., Blaszczynski, A., \& Maccallum, F. (2004). Superstitious beliefs in gambling among problem and non-problem gamblers: Preliminary data. Journal of Gambling Studies, 20, 171-180.

Jöreskog, K. G., \& Sörbom, D. (1996). LISREL 8 user's reference guide. [Scientific Software International]. Uppsala, Sweden.

Khazaal, Y., van Singer, M., Chatton, A., Achab, S., Zullino, D., Rothen, S., ... Thorens, G. (2014). Does self-selection affect samples' representativeness in online surveys? An investigation in online video game research. Journal of Medical Internet Research, 16, e164. http://doi.org/10.2196/jmir.2759

Koob, G. F., \& Le Moal, M. (2008). Addiction and the brain antireward system. Annual Review of Psychology, 59, 29-53. http://doi.org/10.1146/annurev.psych.59.103006.093548

Lambe, L., Mackinnon, S. P., \& Stewart, S. H. (2015). Validation of the gambling motives questionnaire in emerging adults. Journal of Gambling Studies, 31, 867-885. http://doi.org/10.1007/s10899-0149467-0

Lightsey, O. R., \& Hulsey, C. D. (2002). Impulsivity, coping, stress, and problem gambling among university students. Journal of Counseling Psychology, 49, 202-211. http://doi.org/http://dx.doi. org.proxy.bib.ucl.ac.be:888/10.1037/0022-0167.49.2.202 
Lovibond, S. H., \& Lovibond, P. F. (1995). Manual for the depression anxiety stress scale. Sydney: The Psychological Foundation of Australia.

Lyvers, M., Hasking, P., Hani, R., Rhodes, M., \& Trew, E. (2010). Drinking motives, drinking restraint and drinking behaviour among young adults. Addictive Behaviors, 35, 116-122. http:// doi.org/10.1016/j.addbeh.2009.09.011

Mathew, A. R., Wahlquist, A. E., Garrett-Mayer, E., Gray, K. M., Saladin, M. E., \& Carpenter, M. J. (2014). Affective motives for smoking among early stage smokers. Nicotine \& Tobacco Research, 16, 1387-1393. http://doi.org/10.1093/ntr/ntu093

McGrath, D. S., Stewart, S. H., Klein, R. M., \& Barrett, S. P. (2010). Self-generated motives for gambling in two population-based samples of gamblers. International Gambling Studies, 10, 117-138. http:// doi.org/10.1080/14459795.2010.499915

Milosevic, A., \& Ledgerwood, D. M. (2010). The subtyping of pathological gambling: A comprehensive review. Clinical Psychology Review, 30, 988-998. http://doi.org/10.1016/j.cpr.2010.06.013

Neighbors, C., Lostutter, T. W., Cronce, J. M., \& Larimer, M. E. (2002). Exploring college student gambling motivation. Journal of Gambling Studies, 18, 361-370.

Nower, L. \& Blaszczynski, A. (2006). Impulsivity and pathological gambling: A descriptive model. International Gambling Studies, 6, 61-75.

Nunnally, J. C. (1978). Psychometric theory. New York, NY: McGraw-Hill.

Orford, J. (2001). Addiction as excessive appetite. Addiction, 96, 15-31.

Petry, N. M., Stinson, F. S., \& Grant, B. F. (2005). Comorbidity of DSM-IV pathological gambling and other psychiatric disorders: Results From the national epidemiologic survey on alcohol and related conditions. The Journal of Clinical Psychiatry, 66, 564-574. http://doi.org/10.4088/JCP.v66n0504

Raylu, N., \& Oei, T. P. S. (2004). The Gambling Related Cognitions Scale (GRCS): Development, confirmatory factor validation and psychometric properties. Addiction, 99, 757-769. http://doi. org/10.1111/j.1360-0443.2004.00753.x

Schellenberg, B. J. I., McGrath, D. S., \& Dechant, K. (2016). The Gambling Motives Questionnaire financial: Factor structure, measurement invariance, and relationships with gambling behaviour. International Gambling Studies, 16(1), 1-16. http://doi.org/10.1080/14459795.2015.1088559

Stewart, S. H., \& Zack, M. (2008). Development and psychometric evaluation of a three-dimensional Gambling Motives Questionnaire. Addiction, 103, 1110-1117. http://doi.org/10.1111/j.13600443.2008.02235.x

Toneatto, T. (1999). Cognitive psychopathology of problem gambling. Substance Use \& Misuse, 34, 1593-1604.

Toneatto, T., Blitz-Miller, T., Calderwood, K., Dragonetti, R., \& Tsanos, A. (1997). Cognitive distortions in heavy gambling. Journal of Gambling Studies, 13, 253-266.

Wéry, A., \& Billieux, J. (2016). Online sexual activities: An exploratory study of problematic and non-problematic usage patterns in a sample of men. Computers in Human Behavior, 56, 257-266. http://doi.org/10.1016/j.chb.2015.11.046

Wood, R. T. A., \& Griffiths, M. D. (2007). A qualitative investigation of problem gambling as an escape-based coping strategy. Psychology and Psychotherapy: Theory, Research and Practice, 80, 107-125. http://doi.org/10.1348/147608306X107881

Wu, A. M. S., Tao, V. Y. K., Tong, K., \& Cheung, S. F. (2012). Psychometric evaluation of the inventory of Gambling Motives, Attitudes and Behaviours (GMAB) among Chinese gamblers. International Gambling Studies, 12, 331-347. http://doi.org/10.1080/14459795.2012.678273

Yee, N. (2006). The demographics, motivations, and derived experiences of users of massively multiuser online graphical environments. Presence, 15, 309-329. 